\title{
yiGou: A Semantic Text Similarity Computing System Based on SVM
}

\author{
Yang Liu, Chengjie Sun, Lei Lin, and Xiaolong Wang \\ School of Computer Science and Technology \\ Harbin Institute of Technology, China \\ \{yliu, cjsun, linl, wangxl\}@insun.hit.edu.cn
}

\begin{abstract}
This paper describes the yiGou system we developed to compute the semantic similarity of two English sentences, which we submitted to the SemEval 2015 Task 2 (English subtask). The system uses a support vector machine model with literal similarity, shallow syntactic similarity, WordNet-based similarity and latent semantic similarity to predict the semantic similarity score of two short texts. In our experiments, WordNet-based and LSA-based features performed better than other features. Out of the 73 submitted runs, our two runs ranked $38^{\text {th }}$ and $42^{\text {th }}$, with mean Pearson correlation 0.7114 and 0.6964 respectively.
\end{abstract}

\section{Introduction}

Semantic Text Similarity (STS) plays an important role in many Natural language processing tasks, such as Question Answering (Narayanan and Harabagiu, 2004), Machine Translation (Beale et al., 1995), Automatic Summarization (Wang et al., 2008) and Word Sense Disambiguation (Navigli and Velardi, 2005). Since STS is an essential challenge in NLP, that has attracted a significant amount of attention by the research community. SemEval has held tasks about STS for four years in a row, from which we can see the importance and difficulty of this challenge. Particularly, SemEval focuses on semantic similarity of short texts as a lot of researches about long texts have been done in past years and the demand of finding new methods to measure short texts similarity has become stronger in many new applications.

In this paper, we proposed a SVM-based solution to compute the semantic similarity between two sentences which is the goal of SemEval 2015
Task 2. Knowledge-based and corpus-based features were involved in our solution. We used the combination of the word similarity to estimate sentence similarity. And the training data of SemEval 2012 (Agirre et al., 2012) was used to train our model. In our experiments, WordNet-based and LSA-based features performed better than other features. Out of the 73 submitted runs, our two runs ranked $38^{\text {th }}$ and $42^{\text {th }}$, with mean Pearson correlation 0.7114 and 0.6964 respectively. The evaluation results showed that our solution has good generalization ability on the test dataset of SemEval 2015 which is very different from our training set in terms of the sources of the sentences. Some of the relatively new technologies such as Word2Vec (Mikolov et al., 2013) and Sentence2Vec (Le and Mikolov, 2014) are potential methods to represent sentences and will be included in our further works.

\section{Data and Metrics}

In SemEval 2015, the trial dataset comprises the 2012, 2013 and 2014 datasets, which can be used to develop and train models. Because of the limitation of the time, we only used the training data of SemEval 2012 as our training set. The training data of SemEval 2012 contained 2000 sentence pairs from existing paraphrase datasets and machine translation evaluation resources, while the test set of SemEval 2015 coming from image description, news headlines, student answers paired with reference answer, answers to questions posted in stack exchange forums and English discussion forum data exhibiting committed belief. The evaluation metric of SemEval 2015 task 2 is mean Pearson correlation, which is calculated by averaging the Pearson correlations of each subset in the test set. 


\section{Feature engineering}

Considering the training set used in our system, we were trying to generate features which have little relation with the sources where the sentences came from. Four kinds of features are included in our model. They are literal similarity, shallow syntactic similarity, WordNet-based similarity and latent semantic similarity.

\subsection{Literal Similarity}

Intuitively, a pair of sentences that look similar to each other may be similar semantically. For example:

S1: A boy is playing a guitar.

S2: A man is playing a guitar.

S3: Someone is drawing.

Apparently, S1 and S2 look more similar and they are closer in semantics than S1 and S3. We chose the Edit Distance (also known as Levenshtein Distance) over characters to measure the similarity between two sentences. The higher the value is, the less similar the two sentences are. As this measure is case sensitive, we lowercase all letters in the sentences before computing the similarity. Although this method may draw opposite conclusions to our expectations in some specific occasions (For example, I hate it VS I have it, the Edit Distance of this pair of sentences is two, but they express very different meaning), the feature was still kept as we observed that it contributed to the overall performance in our experiments.

\subsection{Shallow Syntactic Similarity}

It is quite a common phenomenon that two sentences only differ in one or two syntactic constituents and have very similar syntactic structures. For example (example comes from training set):

S1: A man is peeling a potato.

S2: A man is slicing a potato.

This pair of sentences got very high score in golden standard file. As we can see, only the predicates of the two sentences are different, and the rest of the sentences are the same. This gives us a clue that using syntactic similarity to build the feature could be feasible. Moreover, two sentences may express exactly the same meaning, but use different English voices. This situation was also considered in our model. Jaccard Distance was chosen to compute this feature, which is defined as follows:

$$
J\left(S_{1}, S_{2}\right)=\frac{\left|S_{1} \cap S_{2}\right|}{\left|S_{1} \cup S_{2}\right|}
$$

Where $S_{1}$ and $S_{2}$ are the collections of Part-OfSpeech tags of each sentence. We used the NLTK toolkit (Bird, 2006) to tag each sentence. Since Jaccard distance measure only cares about the appearance of the tags, and ignores the order of them, it can reduce the impact of the tense change.

\subsection{WordNet-based Similarity}

WordNet (Miller, 1995) is a widely used lexical database for English, and it's a convenient tool to find synonyms of nouns, verbs, adjectives and adverbs. WordNet supports numerous lexical similarity measures (Pedersen et al., 2004). In this work, we explore using two of these similarity measures: res_similarity and path_similarity. The core idea behind the path_similarity measure is that the similarity between two concepts can be derived from the length of the path linking the concepts and the position of the concepts in the WordNet taxonomy. (Meng et al., 2013). While res_similarity (Resnik, 2011) is a similarity measure based on information content. The result of res_similarity is dependent on the corpus that generates the information content.

A girl is slicing a potato.

$0.077 \quad 0.0 \quad 0.33$

A boy is cutting a tomato.

A girl is slicing a potato.

$\begin{array}{lllll}0.167 & 1.0 & 0.33 & 0.33\end{array}$

A boy is cutting a tomato.

Figure 1 An example of word alignment using maximum path_similarity. The upper part of the figure is showing the alignment candidates for tomato scored with path_similarity and the lower part of the figure is showing the max path_similarity alignment for the content words in the sentence pair.

In our system, we used the NLTK WordNet API to compute WordNet-based similarity. Based on WordNet and Brown corpus, the computing of res_similarity and path_similarity involve following steps:

- Partition a pair of sentences into two lists of tokens.

- Part-of-speech tagging.

- Find out the most appropriate sense for every word according to the tagging results; put the 


\begin{tabular}{c|c|c|c|c|c|c}
\hline Features & MSRpar & MSRvid & SMTeuroparl & Sur.OnWN & Sur.SMTnews & Mean \\
\hline All & 0.51237 & 0.83766 & 0.48213 & 0.67070 & 0.47941 & $\mathbf{0 . 5 9 6 4 5 4}$ \\
w/o res_similarity & 0.50939 & 0.83920 & 0.47976 & 0.66406 & 0.47976 & $\mathbf{0 . 5 9 4 4 3 4}$ \\
w/o path_similarity & 0.37667 & 0.78555 & 0.38714 & 0.64145 & 0.45963 & $\mathbf{0 . 5 3 0 0 8 8}$ \\
w/o WN-based sim & 0.37583 & 0.79046 & 0.38930 & 0.64348 & 0.45767 & $\mathbf{0 . 5 3 1 3 4 8}$ \\
\hline
\end{tabular}

Table 1 Results of comparing the importance of res_similarity and path_similarity on test set of SemEval 2012. The WN-based sim included both res_similarity and path_similarity.

\begin{tabular}{c|c|c|c|c|c|c}
\hline Corpus & MSRpar & MSRvid & SMTeuroparl & Sur.OnWN & Sur.SMTnews & Mean \\
\hline Brown & 0.51237 & 0.83766 & 0.48213 & 0.67070 & 0.47941 & $\mathbf{0 . 5 9 6 4 5 4}$ \\
Bnc & 0.51199 & 0.83770 & 0.48157 & 0.66719 & 0.48050 & $\mathbf{0 . 5 9 5 7 9 0}$ \\
Treebank & 0.51199 & 0.83781 & 0.48181 & 0.66689 & 0.48066 & $\mathbf{0 . 5 9 5 8 3 2}$ \\
Semcor & 0.51269 & 0.83768 & 0.48017 & 0.66763 & 0.48017 & $\mathbf{0 . 5 9 5 6 6 8}$ \\
Semcorraw & 0.51274 & 0.83792 & 0.48138 & 0.66691 & 0.47997 & $\mathbf{0 . 5 9 5 7 8 4}$ \\
Shaks & 0.51120 & 0.83746 & 0.48229 & 0.66665 & 0.48105 & $\mathbf{0 . 5 9 5 7 3 0}$ \\
\hline
\end{tabular}

Table 2 Results of using different corpus in res_similarity on test set of SemEval 2012.

results into two lists $S 1$ and $S 2$.

- For every word $w$ in $S 1$, find out the word in $S 2$ that has the maximum res_similarity/ path_similarity with $w$. Adding all of the similarity values together, and then average this value with the length of $S 1$.

- $\quad$ For every word $w$ in $S 2$, find out the word in S1 that has the maximum res_similarity/ path_similarity with $w$. Adding all of the similarity values together, and then average this value with the length of $S 2$.

- Computing the harmonic mean of the two average values, and the result is the value of this feature.

Figure 1 is an example shows how we find the corresponding word which has the maximum res_similarity/path_similarity with the words in the second sentence. In this example, potato has the maximum path_similarity score with tomato, compared to girl and slicing ( 0.33 vs. 0.0077 and 0.0 ). In the bottom part of the figure, each word in the first sentence would find one word which has the maximum similarity score in the second sentence, these scores would then be used to compute this feature.

To compare the importance of the two measures, we separately exclude one of the two features from all the features used in our solution to train two models and compare their performance. The results are shown in Table 1. As we can see from the table, path_similarity contributes more to our overall performance than res_similarity. According to the definition of res_similarity, we changed the corpus to find out the influence of the corpus on our over- all performance. The results are showed in Table 2, from which we can see that the results varied very little with different corpora. In our submitted model, Brown corpus (Francis and Kucera, 1979) was used to compute information content.

\subsection{Latent Semantic Similarity}

All of the features generated above contained little semantic information. While sentences from some sources such as headlines and image descriptions are always have various forms which may not be easily compared through some string match measures or shallow syntactic oriented measures. So, a new feature that measures similarity in semantic space is necessary. Latent semantic analysis (Landauer et al., 1998) is a very popular technique to convert the term-document matrix which describes the occurrences of terms in document into three smaller matrixes like follows:

$$
X=U \Sigma V^{T}
$$

Where $U$ could be preserved as the semantic space of words. Each word could be represented as a row vector in $U$. When measuring semantic similarity of two sentences, all word vectors appeared in the sentence were summed and then averaged with the length of the sentences. Thus we can get vector representations of the two sentences V1 and V2. With $V 1$ and $V 2$, the similarity of the two sentences can be measured with cosine similarity. Cosine similarity defined as follows:

$$
\operatorname{Cos}(V 1, V 2)=\frac{V 1 \cdot V 2}{\|V 1\|\|V 2\|}
$$




\begin{tabular}{c|c|c|c|c|c|c}
\hline Features & MSRpar & MSRvid & SMTeuroparl & Sur.OnWN & Sur.SMTnews & Mean \\
\hline 1 to 2 & -0.05064 & 0.23562 & -0.13259 & 0.07697 & -0.03636 & $\mathbf{0 . 0 1 8 6 0 0}$ \\
1 to 3 & 0.50225 & 0.82813 & 0.41859 & 0.57242 & 0.35525 & $\mathbf{0 . 5 3 5 3 2 8}$ \\
1 to 4 & 0.50593 & 0.82628 & 0.41881 & 0.57676 & 0.35390 & $\mathbf{0 . 5 3 6 3 3 6}$ \\
1 to 5 & 0.51120 & 0.83746 & 0.48229 & 0.66665 & 0.48105 & $\mathbf{0 . 5 9 5 7 3 0}$ \\
1 to 7 & 0.51237 & 0.83766 & 0.48213 & 0.67070 & 0.47941 & $\mathbf{0 . 5 9 6 4 5 4}$ \\
\hline
\end{tabular}

Table 4 Results of SVR on SemEval 2012 test set with different feature combinations.

\begin{tabular}{c|c}
\hline Feature_ID & Feature_Name \\
\hline 1 & EditDistance \\
2 & JaccardDistance \\
3 & path_similarity \\
4 & res_similarity \\
5 & Latent Semantic Similarity \\
6 & IDF-weighted-LSA \\
7 & Freq-weighted-LSA
\end{tabular}

Table 3 All features we used in our submitted model.

In our experiment, we directly used the LSA model provided by SEMILAR ${ }^{1}$. A word is represented as a row vector in the LSA model (Niraula et al., 2014), and the model was decomposed from the whole Wikipedia articles.

We also developed two weighted LSA features to further use semantic information, they are $I D F$ weighted-LSA and Freq-weighted-LSA. IDFweighted-LSA weighted the words (one word is represented as a 200-dimension vector generated from LSA) using inverse document frequency and then summed up all the weighted vectors of words which appeared in the sentence to be the representation of the sentence. The cosine distance of two sentence representations is the value of this feature. Freq-weighted-LSA used word frequency to weight the words and following the same steps mentioned above. In our experiment, the IDF and WordFrequency values were calculated on Wikipedia corpus dumped in December of 2012 (Jin et al., 2014). These features were only included in our second run yiGou-midbaitu. Unfortunately, this system got worse performance than the first run in official estimation. This may be caused by the overfitting of our model on the training data.

\section{Experiments and Results}

Due to the limitation of the time, in our submitted system, we trained Support Vector Regression (SVR) models using Scikit-learn toolkit (Pedrego

\footnotetext{
${ }^{1} \mathrm{http}: / /$ www.semanticsimilarity.org/
}

\begin{tabular}{c|c|c|c|c}
\hline parameter & kernel & gamma & $C$ & epsilon \\
\hline value & rbf & 0.0 & 1.0 & 0.1 \\
\hline \multicolumn{2}{c}{ Table 5 Parameter setting in our models. }
\end{tabular}

sa et al., 2011). Table 3 shows the features used in our submitted models. The results with different feature combinations on the test set of SemEval 2012 are shown in Table 4. Table 5 is our parameter settings.

The performance of the best system in SemEval 2012 is 0.67 (Mean) with 19 features, and our best performance is 0.596 (Mean) with 7 features. In SemEval 2015, out of the 73 submitted runs, our two runs ranked 38th and 42th (with mean Pearson correlation 0.7114 and 0.6964 respectively). And the best performance in 2015 is 0.8015 .

\section{Conclusions and Future Work}

In this paper, we presented our system that participated in the Semantic Text Similarity task in SemEval 2015. We proposed a method using SVR to combine various features to evaluate the similarity between two sentences. We found that WordNet based and LSA-based features are very useful for semantic similarity computing. For future work, we would like to further explore features about semantic representations of words, generate more features related to sentence structures and try to employ some new technologies such as Word2Vec and Sentence2Vec in our model. Besides, using a single model is not adequate to get a better accuracy, other models will be tried and compared in our further work.

\section{Acknowledgments}

The authors would like to thank the SemEval-2015 Task 2 organizers for their hard work. We also thank Daniel Cer and the anonymous reviewers for their helpful suggestions and comments. This work is supported by the National Natural Science Foundation of China (61100094 \& 61300114). 


\section{References}

Eneko Agirre, Mona Diab, Daniel Cer, \& Aitor Gonzalez-Agirre. (2012). Semeval-2012 task 6: A pilot on semantic textual similarity. In Proceedings of the First Joint Conference on Lexical and Computational Semantics-Volume 1: Proceedings of the main conference and the shared task, and Volume 2: Proceedings of the Sixth International Workshop on Semantic Evaluation.

Stephen Beale, Sergei Nirenburg, \& Kavi Mahesh. (1995). Semantic analysis in the Mikrokosmos machine translation project. In Proceedings of the 2nd Symposium on Natural Language Processing.

Steven Bird. (2006). NLTK: the natural language toolkit. In Proceedings of the COLING/ACL on Interactive presentation sessions.

W Nelson Francis, \& Henry Kucera. (1979). Brown corpus manual. Brown University Department of Linguistics.

Pedregosa, F., Varoquaux, G., Gramfort, A., Michel, V., Thirion, B., Grisel, O., ... \& Duchesnay, É. (2011). Scikit-learn: Machine learning in Python. The Journal of Machine Learning Research, 12, 2825 2830.

Meng, L., Huang, R., \& Gu, J. (2013). A review of semantic similarity measures in wordnet. International Journal of Hybrid Information Technology, 6(1), 1-12.

Xiaoqiang Jin, Chengjie Sun, Lei Lin, \& Xiaolong Wang. (2014). Exploiting Multiple Resources for Word-Phrase Semantic Similarity Evaluation Chinese Computational Linguistics and Natural Language Processing Based on Naturally Annotated Big Data (pp. 46-57): Springer.

Thomas K Landauer, Peter W Foltz, \& Darrell Laham. (1998). An introduction to latent semantic analysis. Discourse processes, 25(2-3), 259-284.

Quoc V Le, \& Tomas Mikolov. (2014). Distributed Representations of Sentences and Documents. arXiv preprint arXiv: 1405.4053.

Tomas Mikolov, Kai Chen, Greg Corrado, \& Jeffrey Dean. (2013). Efficient estimation of word representations in vector space. arXiv preprint arXiv:1301.3781.

George A Miller. (1995). WordNet: a lexical database for English. Communications of the ACM, 38(11), 39-41.

Srini Narayanan, \& Sanda Harabagiu. (2004). Question answering based on semantic structures. In Proceedings of the 20th international conference on Computational Linguistics.

Roberto Navigli, \& Paola Velardi. (2005). Structural semantic interconnections: a knowledge-based approach to word sense disambiguation. Pattern
Analysis and Machine Intelligence, IEEE Transactions on, 27(7), 1075-1086.

Nobal B Niraula, Vasile Rus, Rajendra Banjade, Dan Stefanescu, William Baggett, \& Brent Morgan. (2014). The dare corpus: A resource for anaphora resolution in dialogue based intelligent tutoring systems. Proceedings of Language Resources and Evaluation, LREC.

Ted Pedersen, Siddharth Patwardhan, \& Jason Michelizzi. (2004). WordNet:: Similarity: measuring the relatedness of concepts. In Proceedings of the Demonstration Papers at HLT-NAACL 2004.

Philip Resnik. (2011). Semantic similarity in a taxonomy: An information-based measure and its application to problems of ambiguity in natural language. arXiv preprint arXiv: 1105.5444.

Sheldon Ross. (2009). A First Course in Probability 8th Edition: Pearson.

Frane Šarić, Goran Glavaš, Mladen Karan, Jan Šnajder, \& Bojana Dalbelo Bašić. (2012). Takelab: Systems for measuring semantic text similarity. In Proceedings of the First Joint Conference on Lexical and Computational Semantics-Volume 1: Proceedings of the main conference and the shared task, and Volume 2: Proceedings of the Sixth International Workshop on Semantic Evaluation.

Dingding Wang, Tao Li, Shenghuo Zhu, \& Chris Ding. (2008). Multi-document summarization via sentencelevel semantic analysis and symmetric matrix factorization. In Proceedings of the 31st annual international ACM SIGIR conference on Research and development in information retrieval. 